The double-blind, randomized, controlled trial included 33 patients with systemic lupus erythematosus (SLE) and cutaneous lesions. Over a 6 -month period, 16 patients received $100 \mathrm{mg}$ clofazimine and 17 patients received $250 \mathrm{mg}$ chloroquine, both once daily. A stable prednisone dose was administered throughout, as was twice-daily broad-spectrum sunscreen. Two independent, blinded observers assessed the lesions on a scale of 1-6, and SLE disease activity was assessed using the Mexican SLE Disease Activity Index. Patients whose prednisone needed to be increased or who received immunosuppressive drugs were withdrawn from the trial, and the scores obtained at last evaluation were analyzed.

The trial found that $75 \%$ of clofazimine patients and $82.4 \%$ of chloroquine patients responded to treatment, and in both groups improvement was greatest towards the end of the trial. SLE flare caused five clofaziminetreated patients to withdraw from the trial and one patient died; in the chloroquine group, one patient withdrew. Because of a lack of a placebo group and a small number of patients, the possibility that clofazimine caused the SLE flares cannot be ruled out. Long-term efficacy and safety studies are required for clofazimine, but this agent could be an option for treatment of cutaneous lesions in chloroquine-refractory patients with SLE.

Rachel Murphy

Original article Bezerra ELM et al. (2005) Doubleblind, randomized, controlled clinical trial of clofazimine compared with chloroquine in patients with systemic lupus erythematosus. Arthritis Rheum 52: 3073-3078

\section{Targeting interleukin-6 in systemic juvenile idiopathic arthritis}

Interleukin (IL)-6 is a central cytokine in the pathogenesis of systemic juvenile idiopathic arthritis (SJIA) and, according to data from an open-label, single-dose, phase II trial, might represent a promising therapeutic target for this disease.

A total of 18 patients with active SJIA were given a single $2 \mathrm{mg} / \mathrm{kg}, 4 \mathrm{mg} / \mathrm{kg}$ or $8 \mathrm{mg} / \mathrm{kg}$ dose of the humanized anti-human-IL-6-receptor antibody MRA (tocilizumab) by intravenous infusion, in conjunction with background therapy with methotrexate (maximum dose of $20 \mathrm{mg} / \mathrm{m}^{2}$ weekly) in 12 of the participants. No drug-related serious infections were reported for any dose during the infusion or follow-up, and no dose-limiting toxicity or safety issues were reported. A clinical response was seen in all 12 children involved in the efficacy analysis at $48 \mathrm{~h}$ regardless of the dose received and clinical improvement according to the JIA core criteria set (i.e. at least three of six core set criteria improved by $30 \%$ and no more than one worsened by $30 \%$ ) was seen in 11 patients at week 1. Phase III trials are needed to extend these promising observations.

Jenny Buckland

Original article Woo P et al. (2005) Open label phase II trial of single, ascending doses of MRA in Caucasian children with severe systemic juvenile idiopathic arthritis: proof of principle of the efficacy of IL-6 receptor blockade in this type of arthritis and demonstration of prolonged clinical improvement. Arthritis Res Ther 7: R1281-R1288

\section{Tumor necrosis factor antagonists do not increase cancer risk in patients with RA}

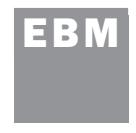

It has been revealed by two Swedish population-based studies that tumor necrosis factor (TNF) antagonists appear not to increase the short-term risk of developing solid cancers or hematopoietic malignancies in patients with rheumatoid arthritis (RA). The studies have also shown that RA patients have an increased risk of hematopoietic malignancies and certain solid cancers compared to the general population.

The two studies, one assessing hematopoietic malignancy and the other assessing solid cancers, each reviewed three cohorts of patients. The inpatient cohort of prevalent RA used data from the Swedish Inpatient Register for 53,067 patients discharged from hospital with a diagnosis of RA between 1990 and 2003. The early cohort of incident RA used information from the Early Arthritis Register for 3,703 patients that had incident (less than 1 year from onset) RA between 1995 and 2003. In the TNF-antagonist cohort, 4,160 patients treated with etanercept, infliximab or adalimumab between 1999 and 2003 were analyzed using data from professionbased surveillance programs. The incidence of cancer in the cohorts was determined by cross-referencing patients' national registration numbers with numbers in the Swedish Cancer Registry and other Swedish national registers. 